\title{
Validity of Thermodynamic Laws and Weak Cosmic Censorship for AdS Black Holes and Black Holes in a Cavity
}

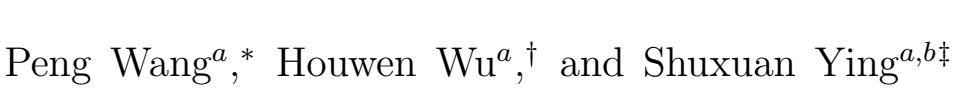 \\ ${ }^{a}$ Center for Theoretical Physics, College of Physics, \\ Sichuan University, Chengdu, 610064, PR China and \\ ${ }^{b}$ Department of Physics, Chongqing University, Chongqing, 401331, China
}

\begin{abstract}
By throwing a test charged particle into a Reissner-Nordstrom (RN) black hole, we test the validity of the first and second laws of thermodynamics and weak cosmic censorship conjecture (WCCC) with two types of boundary conditions, i.e., the asymptotically anti-de Sitter (AdS) space and a Dirichlet cavity wall placed in the asymptotically flat space. For the RN-AdS black hole, the second law of thermodynamics is satisfied, and the WCCC is violated for both extremal and nearextremal black holes. For the RN black hole in a cavity, the entropy can either increase or decrease depending on the change in the charge, and WCCC is satisfied/violated for the extremal/nearextremal black hole. Our results indicate that there may be a connection between the black hole thermodynamics and the boundary condition imposed on the black hole.
\end{abstract}

\footnotetext{
*Electronic address: pengw@scu.edu.cn

${ }^{\dagger}$ Electronic address: iverwu@scu.edu.cn

${ }^{\ddagger}$ Electronic address: ysxuan@cqu.edu.cn
} 


\section{Contents}

I. Introduction

II. RN-AdS Black Hole

III. RN Black Hole in a Cavity

IV. Conclusion

Acknowledgments

References

\section{INTRODUCTION}

Studying thermodynamic properties of black holes can have a deep impact upon the understanding of quantum gravity. Penrose first noticed that a particle can extract energy from a black hole with an ergosphere [1], which led to the discovery of the irreducible mass [2 4]. The square of the irreducible mass of a black hole can be interpreted as the black hole entropy [5, 6]. Later, analogous to the laws of thermodynamics, the four laws of black hole mechanics were proposed [7]. With the advent of the AdS/CFT correspondence [8], there has been much interest in studying thermodynamics and phase structure of AdS black holes [9 22], where some intriguing phase behavior, e.g., reentrant phase transitions and tricritical points, was found to be present. It is also worth pointing out that black holes can become thermally stable in AdS space since the AdS boundary acts as a reflecting wall.

Along with the development of black hole thermodynamics, the weak cosmic censorship conjecture (WCCC) was proposed to hide singularities by event horizons [1]. So if the WCCC is valid, the singularities cannot be seen by the observers at the future null infinity. To test the validity of the WCCC, Wald constructed a gedanken experiment to destroy an extremal Kerr-Newman black hole by overcharging or overspinning the black hole via throwing a test particle into it [23]. Nevertheless, the extremal Kerr-Newman black hole was shown to be incapable of capturing particles with sufficient charge or angular momentum to overcharge or overspin the black hole. Later, near-extremal charged/rotating black holes 
were found to be overcharged/overspun by absorbing a particle [24 26], and hence the WCCC is violated. However, subsequent researches showed that the WCCC might be still valid if the backreaction and self-force effects were considered [27-32]. Since there is still a lack of the general proof of the WCCC, its validity has been tested in various black holes [33-72]. In particular, the thermodynamics and WCCC have been considered for a Reissner-Nordstrom (RN)-AdS black via the charged particle absorption in the normal and extended phase spaces [46, 73]. In the normal phase space, in which the cosmological constant is fixed, it showed that the first and second laws of thermodynamics are satisfied while the WCCC is violated even for an extremal RN-AdS black hole.

Instead of the AdS boundary, York showed that placing Schwarzschild black holes inside a cavity, on the wall of which the metric is fixed, can make them thermally stable [74]. Thermodynamics and phase structure of RN black holes in a cavity were studied in a grand canonical ensemble [75] and a canonical ensemble [76, 77]. It was found that the Schwarzschild and RN black holes in a cavity have quite similar phase structure and transition to these of the AdS counterparts. Afterwards, various black brane systems [78 83], a Gauss-Bonnet black hole [84], hairy black holes [85-88] and Boson stars [89 92] in a cavity were extensively investigated, and it also showed that the behavior of the gravity systems in a cavity is strikingly similar to that of the counterparts in AdS gravity. However, we have recently studied phase structure of Born-Infeld black holes enclosed in a cavity [93, 94] and thermodynamic geometry of RN black holes in a cavity [95], and found their behavior has dissimilarities from that of the corresponding AdS black holes. Note that thermodynamics and critical behavior of de Sitter black holes in a cavity were investigated in [96, 97].

Although there have been a lot of work in progress on the thermodynamic laws and WCCC for various black holes of different theories of gravity in spacetimes with differing asymptotics, little is known about the second law of thermodynamics and WCCC for a black hole enclosed in a cavity. Since RN-AdS black holes and RN black holes in a cavity are thermally stable, they provide appropriate scenarios to explore whether or not the thermodynamic laws and WCCC are sensitive to the boundary condition of black holes. To this end, we study the thermodynamic laws and WCCC for a RN black hole in a cavity in this paper.

The rest of this paper is organized as follows. In section II, we review the discussion of the thermodynamic laws and WCCC for a RN-AdS black hole to be self-contained and introduce the method used in this paper. The thermodynamic laws and WCCC of a RN 
black hole in a cavity are then tested via the absorption of a charged particle in section III. We summarize our results with a brief discussion in section IV. For simplicity, we set $G=\hbar=c=k_{B}=1$ in this paper.

\section{RN-ADS BLACK HOLE}

In this section, we discuss the first and second laws of thermodynamics and WCCC for a RN-AdS black hole by throwing a test particle into the black hole. First, we consider the motion of a test particle of energy $E$, charge $q$ and mass $m$ in a 4-dimensional charged static black hole with the line element,

$$
d s^{2}=-f(r) d t^{2}+\frac{1}{f(r)} d r^{2}+r^{2}\left(d \theta^{2}+\sin ^{2} \theta d \phi^{2}\right),
$$

and the electromagnetic potential $A_{\mu}$,

$$
A_{\mu}=A_{t}(r) \delta_{\mu t} .
$$

We also suppose that the outermost horizon of the black hole is at $r=r_{+}$, where $f\left(r_{+}\right)=0$. In [58], the Hamilton-Jacobi equation of the test particle was given by

$$
-\frac{\left[E+q A_{t}(r)\right]^{2}}{f(r)}+\frac{\left[P^{r}(r)\right]^{2}}{f(r)}+\frac{L^{2}}{r^{2}}=m^{2},
$$

where $L$ and $P^{r}(r)$ are the particle's angular momentum and radial momentum, respectively. It was shown in [98, 99] that $P^{r}\left(r_{+}\right)$is finite and proportional to the Hawking temperature of the black hole. Eqn. (3) gives

$$
E=-q A_{t}(r)+\sqrt{f(r)\left(m^{2}+\frac{L^{2}}{r^{2}}\right)+\left[P^{r}(r)\right]^{2}},
$$

where we choose the positive sign in front of the square root since the energy of the particle is required to be a positive value [2, 4]. At the horizon $r=r_{+}$, the above equation reduces to

$$
E=q \Phi+\left|P^{r}\left(r_{+}\right)\right|,
$$

where $\Phi \equiv-A_{t}\left(r_{+}\right)$is the electric potential of the black hole. Eqn. (5) relates the energy of the particle to its radial momentum and potential energy just before the particle enters the horizon. 
To check whether a particle can reach or exist near the black hole horizon, we can rewrite eqn. (4) as the radial equation of motion,

$$
\left(\frac{d r}{d \tau}\right)^{2}=\frac{\left[E+q A_{t}(r)\right]^{2}}{m^{2}}-f(r)\left(1+\frac{L^{2}}{m^{2} r^{2}}\right)
$$

where we use $P^{r}(r)=m d r / d \tau$, and $\tau$ is the affine parameter along the worldline. Note that a particle can exist in the region where $(d r / d \tau)^{2} \geq 0$, and $d r / d \tau=0$ corresponds to a turning point. Specifically for a particle existing at the event horizon, eqn. (6) gives that $\left.(d r / d \tau)^{2}\right|_{r=r_{+}} \geq 0$, and hence $E \geq q \Phi$. Furthermore, if the particle falls into the black hole, one has $(d r / d \tau)^{2}>0$ at the event horizon, which leads to $E>q \Phi$. In short, for a particle of energy $E$ and charge $q$ around a black hole of potential $\Phi, E>q \Phi$ provides a lower bound $E_{\text {low }}$ on $E$, which ensures that the particle exists near the event horizon and gets absorbed by the black hole.

For a RN-AdS black hole, the metric function $f(r)$ and electric potential $\Phi$ are

$$
f(r ; M, Q)=1-\frac{2 M}{r}+\frac{Q^{2}}{r^{2}}+\frac{r^{2}}{l^{2}} \text { and } \Phi=\frac{Q}{r_{+}},
$$

respectively, where $M$ and $Q$ are the mass and charge of the black hole, respectively, and $l$ is the AdS radius. Here the parameters $M$ and $Q$ are put explicitly as arguments of the function $f(r)$ for later convenience. With fixed charge $Q$, the mass $M_{e}(Q)$ and horizon radius $r_{e}(Q)$ of the extremal $\mathrm{RN}$-AdS black hole are determined by $f\left(r_{e}(Q) ; M, Q\right)=$ $d f(r ; M, Q) /\left.d r\right|_{r=r_{e}(Q)}=0$, which gives

$$
\begin{aligned}
& M_{e}(Q)=\frac{\sqrt{6} l}{18}\left(2+\sqrt{1+12 Q^{2} / l^{2}}\right) \sqrt{\sqrt{1+12 Q^{2} / l^{2}}-1}, \\
& r_{e}(Q)=\frac{l}{\sqrt{6}} \sqrt{\sqrt{1+12 Q^{2} / l^{2}}-1} .
\end{aligned}
$$

Like a RN black hole, when $M \geq M_{e}(Q)$, the RN-AdS black hole solution possesses the event horizon at $r=r_{+}(M, Q)$, which is obtained by solving $f(r ; M, Q)=0$ [73]. Otherwise, the event horizon disappears, and a naked singularity emerges, which leads to the violation of the WCCC. If the event horizon exists, one can define the black hole's temperature $T$ and entropy $S$ as

$$
T=\left.\frac{1}{4 \pi} \frac{\partial f(r ; M, Q)}{\partial r}\right|_{r=r_{+}(M, Q)} \text { and } S=\pi r_{+}^{2}(M, Q) .
$$

Suppose one starts with an initial black hole of mass $M$ and charge $Q$ with $M \geq M_{e}(Q)$ and throws a test particle of energy $E \ll M$ and charge $q \ll Q$ into the black hole. After 
the particle is absorbed, the final configuration has mass $M+d M$ and charge $Q+d Q$. The energy and charge conservation of the absorbing process gives

$$
d M=E \text { and } d Q=q
$$

where $E$ and $q$ are related via eqn. (5). If $M+E \geq M_{e}(Q+q)$, there exits an event horizon in the final black hole solution, which hides the naked singularity. However for $M+E<$ $M_{e}(Q+q)$, the naked singularity can be seen by distant observers due to the absence of the event horizon.

We first check the first and second laws of thermodynamics for a RN-AdS black hole during the absorption. In this case, the final black hole solution should possess an event horizon at $r=r_{+}(M, Q)+d r_{+}$in order that thermodynamic variables are well defined. So the horizon radius, mass and charge of the final black hole satisfy

$$
f\left(r_{+}(M, Q)+d r_{+} ; M+d M, Q+d Q\right)=0
$$

which leads to

$$
\left.\frac{\partial f(r ; M, Q)}{\partial r}\right|_{r=r_{+}(M, Q)} d r_{+}+\left.\frac{\partial f(r ; M, Q)}{\partial M}\right|_{r=r_{+}(M, Q)} d M+\left.\frac{\partial f(r ; M, Q)}{\partial Q}\right|_{r=r_{+}(M, Q)} d Q=0 .
$$

Using eqns. (5), (7), (9) and (10), one finds that the above equation gives

$$
\left|P^{r}\left(r_{+}(M, Q)\right)\right|=T d S
$$

For an extremal black hole with $T=0$, since $P^{r}\left(r_{+}(M, Q)\right) \propto T$, eqn. (13) is trivial. Nevertheless, for a non-extremal RN-AdS black hole with $T>0$, the variation of entropy is

$$
d S=\frac{\left|P^{r}\left(r_{+}(M, Q)\right)\right|}{T}>0
$$

which means the second law of thermodynamics is satisfied. Moreover, plugging eqn. (10) and (13) into eqn. (5) yields the first law of thermodynamics:

$$
d M=\Phi d Q+T d S
$$

To test the WCCC, we consider an extremal or near-extremal RN-AdS black hole and check whether throwing a charged particle can overcharge the black hole. To overcharge the black hole, the final configuration should exceeds extremality:

$$
M+E<M_{e}(Q+q),
$$


which, together with eqn. (5), gives the constraints on the energy of the particle

$$
E_{\mathrm{low}} \equiv \frac{q Q}{r_{+}(M, Q)}<E<M_{e}(Q+q)-M \equiv E_{\mathrm{up}}
$$

Since $q \ll Q$, we can expand $M_{e}(Q+q)$ and obtain

$$
E_{\mathrm{up}} \simeq M_{e}(Q)-M+M_{e}^{\prime}(Q) q+\frac{M_{e}^{\prime \prime}(Q)}{2} q^{2}
$$

where

$$
M_{e}^{\prime}(Q)=\frac{Q}{r_{e}(Q)} \text { and } M_{e}^{\prime \prime}(Q)=\frac{\sqrt{\sqrt{1+12 Q^{2} / l^{2}}-1}}{l \sqrt{2 / 3+8 Q^{2} / l^{2}}}>0 .
$$

If the initial black hole is extremal, the lower and upper bounds on $E$ become

$$
E_{\mathrm{low}}=\frac{q Q}{r_{e}(Q)} \text { and } E_{\mathrm{up}} \simeq M_{e}^{\prime}(Q) q+\frac{M_{e}^{\prime \prime}(Q)}{2} q^{2}>E_{\mathrm{low}} .
$$

So there always exists a test changed particle with $E_{\text {low }}<E<E_{\text {up }}$, which can overcharge the extremal RN-AdS black hole. For a near-extremal RN-AdS black hole with $Q$ and $M=M_{e}(Q)+\epsilon^{2}$, the lower and upper bounds on $E$ become

$$
E_{\mathrm{low}} \simeq M_{e}^{\prime}(Q) q-A(Q) q \epsilon \text { and } E_{\mathrm{up}} \simeq M_{e}^{\prime}(Q) q+\frac{M_{e}^{\prime \prime}(Q)}{2} q^{2}-\epsilon^{2},
$$

where $A(Q)>0$ is some function of $Q$, and $\epsilon$ is a small parameter. To have $E_{\text {up }}>E_{\text {low }}$, we find

$$
q \equiv a \epsilon>\frac{-A(Q)+\sqrt{A^{2}(Q)+2 M_{e}^{\prime \prime}(Q)}}{M_{e}^{\prime \prime}(Q)} \epsilon .
$$

The constraints (17) gives the energy $E$ of the particle,

$$
E=M_{e}^{\prime}(Q) a \epsilon+b \epsilon^{2} \text { with }-A(Q) a<b<\frac{M_{e}^{\prime \prime}(Q)}{2} a^{2}-1
$$

Therefore, a charged particle with its charge and energy satisfying eqns. (22) and (23), respectively, can overcharge the near-extremal black hole. In summary, WCCC is always violated for extremal and near-extremal RN-AdS black holes.

\section{RN BLACK HOLE IN A CAVITY}

In this section, we throw a charged particle into a $\mathrm{RN}$ black hole enclosed in a cavity and test the first and second laws of thermodynamics and WCCC. We now consider a 
thermodynamic system with a RN black hole enclosed in a cavity, the wall of which is at $r=r_{B}$. The 4-dimensional RN black hole solution is

$$
\begin{aligned}
d s^{2} & =-f(r ; M, Q) d t^{2}+\frac{d r^{2}}{f(r ; M, Q)}+r^{2}\left(d \theta^{2}+\sin ^{2} \theta d \phi^{2}\right), \\
f(r ; M, Q) & =1-\frac{2 M}{r}+\frac{Q^{2}}{r^{2}}, A=A_{t}(r) d t=-\frac{Q}{r} d t
\end{aligned}
$$

where $M$ and $Q$ are the black hole charge and mass, respectively. The Hawking temperature $T_{\mathrm{BH}}$ of the black hole is given by

$$
T_{\mathrm{BH}}=\left.\frac{1}{4 \pi} \frac{\partial f(r ; M, Q)}{\partial r}\right|_{r=r_{+}(M, Q)}=\frac{1}{4 \pi r_{+}(M, Q)}\left(1-\frac{Q^{2}}{r_{+}^{2}(M, Q)}\right),
$$

where $r_{+}(M, Q)=M+\sqrt{M^{2}-Q^{2}}$ is the radius of the outer event horizon. Suppose that the wall of the cavity is maintained at a temperature of $T$. It was shown in [75] that the system temperature $T$ can be related to the black hole temperature $T_{\mathrm{BH}}$ as

$$
T=\frac{T_{\mathrm{BH}}}{\sqrt{f\left(r_{B} ; M, Q\right)}}
$$

which means that $T$, measured at $r=r_{B}$, is blueshifted from $T_{\mathrm{BH}}$, measured at $r=\infty$. The thermal energy $\mathcal{E}$ and potential $\Phi$ of this system were [75]

$$
\begin{aligned}
& \mathcal{E}=r_{B}\left[1-\sqrt{f\left(r_{B} ; M, Q\right)}\right], \\
& \Phi=\frac{A_{t}\left(r_{B}\right)-A_{t}\left(r_{+}\right)}{\sqrt{f\left(r_{B} ; M, Q\right)}} .
\end{aligned}
$$

The physical space of $r_{+}(M, Q)$ is bounded by

$$
r_{e}(Q) \leq r_{+}(M, Q) \leq r_{B}
$$

where $r_{e}(Q)=Q$ is the horizon radius of the extremal black hole.

After we throw a particle of energy $E$ and charge $q$ into the RN black hole, the thermal energy and charge of the system are changed from $(\mathcal{E}, Q)$ to $(\mathcal{E}+d \mathcal{E}, Q+d Q)$. The energy and charge conservation give

$$
\begin{aligned}
& d Q=q, \\
& d \mathcal{E}=\frac{1}{\sqrt{f\left(r_{B} ; M, Q\right)}}\left(d M-\frac{Q d Q}{r_{B}}\right)=E,
\end{aligned}
$$


where we use eqn. (27) to express $d \mathcal{E}$ in terms of $d M$ and $d Q$. Here we assume that the radius $r_{B}$ of the cavity is fixed during the absorption. Eqn. (29) leads to the variation of the black hole mass $M$,

$$
d M=\sqrt{f\left(r_{B} ; M, Q\right)} E+\frac{q Q}{r_{B}} .
$$

To discuss the thermodynamic laws, the final RN black hole after absorbing the particle is assumed to possess an event horizon, which is located at $r=r_{+}(M, Q)+d r_{+}$. Similar to the RN-AdS case, we have

$$
\left.\frac{\partial f(r ; M, Q)}{\partial r}\right|_{r=r_{+}(M, Q)} d r_{+}+\left.\frac{\partial f(r ; M, Q)}{\partial M}\right|_{r=r_{+}(M, Q)} d M+\left.\frac{\partial f(r ; M, Q)}{\partial Q}\right|_{r=r_{+}(M, Q)} d Q=0 .
$$

Eqns. (51), (25), (26) and (30) then give

$$
T d S=\left|P^{r}\left(r_{+}(M, Q)\right)\right|+\frac{q Q}{r_{+}(M, Q)}-q \Phi
$$

where $S=\pi r_{+}^{2}(M, Q)$ is the entropy of the system. Using eqn. (27), we rewrite eqn. (32) as

$$
T d S=\left|P^{r}\left(r_{+}(M, Q)\right)\right|+\left(\frac{1}{r_{+}}-\frac{1}{r_{+} \sqrt{f\left(r_{B} ; M, Q\right)}}+\frac{1}{r_{B} \sqrt{f\left(r_{B} ; M, Q\right)}}\right) Q d Q,
$$

where the prefactor of $Q d Q$ is positive. It can show that, when $T=0$ (i.e., $Q=M$ ), both sides of (33) are zero, which cannot provide any information about $d S$. For a non-extremal black hole, eqn. (33) gives that the entropy increases when $d Q>0$. However when $d Q<0$, the entropy can increases or decrease depending the value of $d Q$. So the second law of thermodynamics is indefinite for a RN black hole in a cavity. Substituting eqn. (5) into eqn. (32), we obtain

$$
d \mathcal{E}=\Phi d Q+T d S
$$

which is the first law of thermodynamics.

To overcharge a RN black hole of mass $M$ and charge $Q$ in a cavity by a test particle of energy $E$ and charge $q$, the mass $M+d M$ and charge $Q+q$ of the final configuration must satisfy

$$
M+d M<Q+q
$$

which, due to eqn. (30), puts an upper bound on $E$,

$$
E<E_{\text {up }} \equiv \frac{Q+q-M-\frac{q Q}{r_{B}}}{\sqrt{f\left(r_{B} ; M, Q\right)}}
$$


On the other hand, eqn. (5) also puts a lower bound on $E$,

$$
E>\frac{q Q}{r_{+}(M, Q)} \equiv E_{\text {low }}
$$

For an extremal black hole with $M=Q$, we find

$$
E_{\text {up }}=E_{\text {low }}=q,
$$

which indicates that the extremal black hole cannot be overcharged. Considering a nearextremal RN black hole with $Q$ and $M=Q+\epsilon^{2}$, one can show that

$$
E_{\mathrm{low}} \simeq q-\frac{\sqrt{2} q \epsilon}{\sqrt{Q}} \text { and } E_{\mathrm{up}} \simeq q-\frac{\epsilon^{2}}{1-Q / r_{B}} .
$$

If $E_{\mathrm{up}}>E_{\text {low }}$, the charge $q$ of the test particle should be bounded from below,

$$
q \equiv a \epsilon>\frac{\epsilon}{\left(1-Q / r_{B}\right)} \sqrt{\frac{Q}{2}} .
$$

Moreover, the corresponding energy $E$ of the particle is

$$
E=a \epsilon+b \epsilon^{2} \text { with }-\frac{\sqrt{2} a}{\sqrt{Q}}<b<-\frac{1}{1-Q / r_{B}} .
$$

So a test particle with $(q, E)$ in the parameter region (40) and (41) can overcharge the near-extremal RN black hole in a cavity, which invalidates the WCCC.

\section{CONCLUSION}

In this paper, via absorbing a test charged particle, we calculated the variations of thermodynamic quantities of RN black holes with two boundary conditions, namely the asymptotically AdS boundary and the Dirichlet boundary in the asymptotically flat spacetime. With these variations, we checked the first and second laws of thermodynamics and WCCC in these two cases. Our results are summarized in Table [1 In the limit of $l \rightarrow \infty$, a RN-AdS black hole becomes a RN black hole. Similarly, a RN black hole in a cavity also reduces to a RN black hole when $r_{B} \rightarrow \infty$. We find that taking the limits of our results in both AdS and cavity cases gives the same result about the validity of the thermodynamic laws and WCCC for a RN black hole, which is also presented in Table I.

In [76, 77], thermodynamic and phase structure of a RN-AdS black hole and a RN black hole in a cavity were shown to be strikingly similar. However, it was found that thermodynamic geometry in these two cases behaves rather differently [95], which implies that there 


\begin{tabular}{|l|l|l|l|}
\hline & RN-AdS BH & RN BH in a cavity & RN BH \\
\hline 1st Law & Satisfied. & Satisfied. & Satisfied. \\
\hline 2nd Law & Satisfied. & $\begin{array}{l}\text { Satisfied for } d Q>0 . \quad \text { Indefi- } \\
\text { nite for } d Q<0 .\end{array}$ & Satisfied. \\
\hline WCCC & $\begin{array}{l}\text { Violated for extremal } \\
\text { and near-extremal BHs. }\end{array}$ & $\begin{array}{l}\text { Satisfied for extremal BH. Vi- } \\
\text { olated for near-extremal BH. }\end{array}$ & Satisfied for extremal BH. Vi- \\
\hline
\end{tabular}

TABLE I: Results for the first and second laws of thermodynamics and weak cosmic censorship conjectures (WCCC), which are tested for a RN-AdS black hole, a RN black hole in a cavity and a RN black hole by absorbing a test charged particle.

may be a connection between the black hole microstates and the boundary condition. In this paper, we showed that the validity of the second law of thermodynamic and WCCC in the AdS and cavity cases are quite different, which further motivates us to explore the connection between the internal microstructure of black holes and the boundary condition.

To understand the scale of energy required for a particle to overcharge a black hole, we can convert physical quantities in Plack units to SI units. In fact, for a particle of charge $q$ and energy $E$ in Plack units, the charge and the energy in SI units are $q q_{p}$ and $E m_{p} c^{2}$, respectively, where $q_{p} \equiv \sqrt{4 \pi \epsilon_{0} \hbar c}=e / \sqrt{\alpha}$ is the Planck charge, $\epsilon_{0}$ is the permittivity of free space, $e$ is the elementary charge, $\alpha$ is the fine structure constant, and $m_{p}=\sqrt{\hbar c / G}$ is the Planck mass. From eqns. (22), (23), (40) and (41), we find that a test particle that can overcharge a charged black hole should have

$$
E \sim q(\text { in Planck units) }
$$

which leads to

$$
E \sim \frac{|q|}{e}\left(\sqrt{\alpha} m_{p} c^{2}\right) \sim \frac{|q|}{e} \times 10^{18} \mathrm{GeV} \text { (in SI units) }
$$

For example, if one throws an ionized Hydrogen nuclei of $m \sim 1 \mathrm{GeV} / c^{2}$ and $q=e$ to overcharge a charged black hole, the nuclei is ultrarelativistic with an enormous kinetic energy $\sim 10^{18} \mathrm{GeV}$ to overcome the electrostatic repulsion between the particle and the black hole.

In this paper, we discussed the WCCC in the test limit, in that the interaction between test particles and the black hole background is neglected. Although this method is simple 
and straightforward, it is subject to several limitations. For example, our results showed that the final mass of the black hole needs to have a second-order correction in $q$ to overcharge the black hole. However, if one calculates the mass consistently to order $q^{2}$, the test limit, which is valid only to linear order in $q$, is not enough, and hence all second-order effects, e.g., self-force and finite size effects, should be considered. In [43], a general formula for the full second-order correction to mass was proposed, and it was found that the WCCC is valid for Kerr-Newman black holes up to the second-order perturbation of the matter fields. In this new version of the gedanken experiment, the WCCC was tested and found to be valid for various black holes [47, 48, 100, 101]. In particular, the WCCC was investigated for RN-AdS black holes in the extended phase space in [102], which showed that the WCCC cannot be violated for RN-AdS black holes under the second-order approximation of the matter field perturbations. On the other hand, we used the test limit to study the WCCC for RN-AdS black holes in the normal phase space with fixed cosmological constant, and found that the WCCC is violated. Apart from different phase spaces considered, differences between our results and these in [102] suggest that corrections beyond the test limit can play an important role in the analysis of the WCCC for charged AdS black holes and black holes in a cavity. We leave this for future work.

\section{Acknowledgments}

We are grateful to Haitang Yang and Zhipeng Zhang for useful discussions and valuable comments. This work is supported in part by NSFC (Grant No. 11875196, 11375121 and $11005016)$.

[1] R. Penrose, "Gravitational collapse: The role of general relativity," Riv. Nuovo Cim. 1, 252 (1969) [Gen. Rel. Grav. 34, 1141 (2002)].

[2] D. Christodoulou, "Reversible and irreversible transforations in black hole physics," Phys. Rev. Lett. 25, 1596 (1970). doi:10.1103/PhysRevLett.25.1596

[3] J. M. Bardeen, "Kerr Metric Black Holes," Nature 226, 64 (1970). doi:10.1038/226064a0 
[4] D. Christodoulou and R. Ruffini, "Reversible transformations of a charged black hole," Phys. Rev. D 4, 3552 (1971). doi:10.1103/PhysRevD.4.3552

[5] J. D. Bekenstein, "Black holes and the second law," Lett. Nuovo Cim. 4, 737 (1972). doi:10.1007/BF02757029

[6] J. D. Bekenstein, "Black holes and entropy," Phys. Rev. D 7, 2333 (1973). doi:10.1103/PhysRevD.7.2333

[7] J. M. Bardeen, B. Carter and S. W. Hawking, "The Four laws of black hole mechanics," Commun. Math. Phys. 31, 161 (1973). doi:10.1007/BF01645742

[8] J. M. Maldacena, "The Large N limit of superconformal field theories and supergravity," Int. J. Theor. Phys. 38, 1113 (1999) [Adv. Theor. Math. Phys. 2, 231 (1998)] doi:10.1023/A:1026654312961, 10.4310/ATMP.1998.v2.n2.a1 hep-th/9711200].

[9] A. Chamblin, R. Emparan, C. V. Johnson and R. C. Myers, "Charged AdS black holes and catastrophic holography," Phys. Rev. D 60, 064018 (1999) doi:10.1103/PhysRevD.60.064018 hep-th/9902170.

[10] A. Chamblin, R. Emparan, C. V. Johnson and R. C. Myers, "Holography, thermodynamics and fluctuations of charged AdS black holes," Phys. Rev. D 60, 104026 (1999) doi:10.1103/PhysRevD.60.104026 hep-th/9904197.

[11] M. M. Caldarelli, G. Cognola and D. Klemm, "Thermodynamics of Kerr-Newman-AdS black holes and conformal field theories," Class. Quant. Grav. 17, 399 (2000) doi:10.1088/02649381/17/2/310 hep-th/9908022.

[12] R. G. Cai, "Gauss-Bonnet black holes in AdS spaces," Phys. Rev. D 65, 084014 (2002) doi:10.1103/PhysRevD.65.084014 hep-th/0109133.

[13] D. Kubiznak and R. B. Mann, "P-V criticality of charged AdS black holes," JHEP 1207, 033 (2012) doi:10.1007/JHEP07(2012)033 [arXiv:1205.0559 [hep-th]].

[14] S. Gunasekaran, R. B. Mann and D. Kubiznak, "Extended phase space thermodynamics for charged and rotating black holes and Born-Infeld vacuum polarization," JHEP 1211, 110 (2012) doi:10.1007/JHEP11(2012)110 [arXiv:1208.6251 [hep-th]].

[15] S. W. Wei and Y. X. Liu, "Critical phenomena and thermodynamic geometry of charged Gauss-Bonnet AdS black holes," Phys. Rev. D 87, no. 4, 044014 (2013) doi:10.1103/PhysRevD.87.044014 [arXiv:1209.1707 [gr-qc]].

[16] R. G. Cai, L. M. Cao, L. Li and R. Q. Yang, "P-V criticality in the extended 
phase space of Gauss-Bonnet black holes in AdS space," JHEP 1309, 005 (2013) doi:10.1007/JHEP09(2013)005 [arXiv:1306.6233 [gr-qc]].

[17] W. Xu and L. Zhao, "Critical phenomena of static charged AdS black holes in conformal gravity," Phys. Lett. B 736, 214 (2014) doi:10.1016/j.physletb.2014.07.019 arXiv:1405.7665 [gr-qc]].

[18] A. M. Frassino, D. Kubiznak, R. B. Mann and F. Simovic, "Multiple Reentrant Phase Transitions and Triple Points in Lovelock Thermodynamics," JHEP 1409, 080 (2014) doi:10.1007/JHEP09(2014)080 arXiv:1406.7015 [hep-th]].

[19] M. H. Dehghani, S. Kamrani and A. Sheykhi, " $P-V$ criticality of charged dilatonic black holes," Phys. Rev. D 90, no. 10, 104020 (2014) doi:10.1103/PhysRevD.90.104020 arXiv:1505.02386 [hep-th]].

[20] R. A. Hennigar, W. G. Brenna and R. B. Mann, "P-v criticality in quasitopological gravity," JHEP 1507, 077 (2015) doi:10.1007/JHEP07(2015)077 [arXiv:1505.05517 [hep-th]].

[21] P. Wang, H. Wu and H. Yang, "Thermodynamics and Phase Transitions of Nonlinear Electrodynamics Black Holes in an Extended Phase Space," JCAP 1904, 052 (2019) doi:10.1088/1475-7516/2019/04/052 [arXiv:1808.04506 [gr-qc]].

[22] D. Wu, P. Wu, H. Yu and S. Q. Wu, "Notes on the thermodynamics of superentropic AdS black holes," Phys. Rev. D 101, no. 2, 024057 (2020) doi:10.1103/PhysRevD.101.024057

[23] R. Wald, "Gedanken experiments to destroy a black hole," Ann. Phys. 82, 548 (1974) doi:10.1016/0003-4916(74)90125-0

[24] V. E. Hubeny, "Overcharging a black hole and cosmic censorship," Phys. Rev. D 59, 064013 (1999) doi:10.1103/PhysRevD.59.064013 gr-qc/9808043.

[25] T. Jacobson and T. P. Sotiriou, "Over-spinning a black hole with a test body," Phys. Rev. Lett. 103, 141101 (2009) Erratum: [Phys. Rev. Lett. 103, 209903 (2009)] doi:10.1103/PhysRevLett.103.209903， 10.1103/PhysRevLett.103.141101 arXiv:0907.4146 $[\mathrm{gr}-\mathrm{qc}]]$.

[26] A. Saa and R. Santarelli, "Destroying a extremal Kerr-Newman black hole," Phys. Rev. D 84, 027501 (2011) doi:10.1103/PhysRevD.84.027501 [arXiv:1105.3950 [gr-qc]].

[27] S. Hod, "Weak Cosmic Censorship: As Strong as Ever," Phys. Rev. Lett. 100, 121101 (2008) doi:10.1103/PhysRevLett.100.121101 [arXiv:0805.3873 [gr-qc]].

[28] E. Barausse, V. Cardoso and G. Khanna, "Test bodies and naked singulari- 
ties: Is the self-force the cosmic censor?," Phys. Rev. Lett. 105, 261102 (2010) doi:10.1103/PhysRevLett.105.261102 [arXiv:1008.5159 [gr-qc]].

[29] E. Barausse, V. Cardoso and G. Khanna, "Testing the Cosmic Censorship Conjecture with point particles: the effect of radiation reaction and the self-force," Phys. Rev. D 84, 104006 (2011) doi:10.1103/PhysRevD.84.104006 [arXiv:1106.1692 [gr-qc]].

[30] P. Zimmerman, I. Vega, E. Poisson and R. Haas, "Self-force as a cosmic censor," Phys. Rev. D 87, no. 4, 041501 (2013) doi:10.1103/PhysRevD.87.041501 [arXiv:1211.3889 [gr-qc]].

[31] M. Colleoni and L. Barack, "Overspinning a Kerr black hole: the effect of self-force," Phys. Rev. D 91, 104024 (2015) doi:10.1103/PhysRevD.91.104024 [arXiv:1501.07330 [gr-qc]].

[32] M. Colleoni, L. Barack, A. G. Shah and M. van de Meent, "Self-force as a cosmic censor in the Kerr overspinning problem," Phys. Rev. D 92, no. 8, 084044 (2015) doi:10.1103/PhysRevD.92.084044 arXiv:1508.04031 [gr-qc]].

[33] G. E. A. Matsas and A. R. R. da Silva, "Overspinning a nearly extreme charged black hole via a quantum tunneling process," Phys. Rev. Lett. 99, 181301 (2007) doi:10.1103/PhysRevLett.99.181301 [arXiv:0706.3198 [gr-qc]].

[34] M. Richartz and A. Saa, "Overspinning a nearly extreme black hole and the Weak Cosmic Censorship conjecture,” Phys. Rev. D 78, 081503 (2008) doi:10.1103/PhysRevD.78.081503 arXiv:0804.3921 [gr-qc]].

[35] S. Isoyama, N. Sago and T. Tanaka, "Cosmic censorship in overcharging a ReissnerNordstróm black hole via charged particle absorption," Phys. Rev. D 84, 124024 (2011) doi:10.1103/PhysRevD.84.124024 [arXiv:1108.6207 [gr-qc]].

[36] S. Gao and Y. Zhang, "Destroying extremal Kerr-Newman black holes with test particles," Phys. Rev. D 87, no. 4, 044028 (2013) doi:10.1103/PhysRevD.87.044028 arXiv:1211.2631 [gr-qc]].

[37] S. Hod, "Cosmic Censorship: Formation of a Shielding Horizon Around a Fragile Horizon," Phys. Rev. D 87, no. 2, 024037 (2013) doi:10.1103/PhysRevD.87.024037 arXiv:1302.6658 [gr-qc]].

[38] K. Duztas and I. Semiz, "Cosmic Censorship, Black Holes and Integer-spin Test Fields," Phys. Rev. D 88, no. 6, 064043 (2013) doi:10.1103/PhysRevD.88.064043 arXiv:1307.1481 [gr-qc]].

[39] H. M. Siahaan, "Destroying Kerr-Sen black holes," Phys. Rev. D 93, no. 6, 064028 (2016) 
doi:10.1103/PhysRevD.93.064028 arXiv:1512.01654 [gr-qc]].

[40] J. Natario, L. Queimada and R. Vicente, "Test fields cannot destroy extremal black holes," Class. Quant. Grav. 33, no. 17, 175002 (2016) doi:10.1088/0264-9381/33/17/175002 arXiv:1601.06809 [gr-qc]].

[41] K. Duztas, "Overspinning BTZ black holes with test particles and fields," Phys. Rev. D 94, no. 12, 124031 (2016) doi:10.1103/PhysRevD.94.124031 [arXiv:1701.07241 [gr-qc]].

[42] K. S. Revelar and I. Vega, "Overcharging higher-dimensional black holes with point particles," Phys. Rev. D 96, no. 6, 064010 (2017) doi:10.1103/PhysRevD.96.064010 arXiv:1706.07190 [gr-qc]].

[43] J. Sorce and R. M. Wald, "Gedanken experiments to destroy a black hole. II. Kerr-Newman black holes cannot be overcharged or overspun," Phys. Rev. D 96, no. 10, 104014 (2017) doi:10.1103/PhysRevD.96.104014 arXiv:1707.05862 [gr-qc]].

[44] V. Husain and S. Singh, "Penrose inequality in anti-de Sitter space," Phys. Rev. D 96, no. 10, 104055 (2017) doi:10.1103/PhysRevD.96.104055 [arXiv:1709.02395 [gr-qc]].

[45] T. Crisford, G. T. Horowitz and J. E. Santos, "Testing the Weak Gravity - Cosmic Censorship Connection," Phys. Rev. D 97, no. 6, 066005 (2018) doi:10.1103/PhysRevD.97.066005 arXiv:1709.07880 [hep-th]].

[46] B. Gwak, "Thermodynamics with Pressure and Volume under Charged Particle Absorption," JHEP 1711, 129 (2017) doi:10.1007/JHEP11(2017)129 arXiv:1709.08665 [gr-qc]].

[47] J. An, J. Shan, H. Zhang and S. Zhao, "Five-dimensional Myers-Perry black holes cannot be overspun in gedanken experiments," Phys. Rev. D 97, no. 10, 104007 (2018) doi:10.1103/PhysRevD.97.104007 [arXiv:1711.04310 [hep-th]].

[48] B. Ge, Y. Mo, S. Zhao and J. Zheng, "Higher-dimensional charged black holes cannot be over-charged by gedanken experiments," Phys. Lett. B 783, 440 (2018) doi:10.1016/j.physletb.2018.07.015 arXiv:1712.07342 [hep-th]].

[49] T. Y. Yu and W. Y. Wen, "Cosmic censorship and Weak Gravity Conjecture in the EinsteinMaxwell-dilaton theory," Phys. Lett. B 781, 713 (2018) doi:10.1016/j.physletb.2018.04.060 arXiv:1803.07916 [gr-qc]].

[50] B. Gwak, "Weak Cosmic Censorship Conjecture in Kerr-(Anti-)de Sitter Black Hole with Scalar Field," JHEP 1809, 081 (2018) doi:10.1007/JHEP09(2018)081 arXiv:1807.10630] [gr$\mathrm{qc}]]$. 
[51] Y. Gim and B. Gwak, "Charged black hole in gravity's rainbow: Violation of weak cosmic censorship," Phys. Lett. B 794, 122 (2019) doi:10.1016/j.physletb.2019.05.039 arXiv:1808.05943 [gr-qc]].

[52] D. Chen, "Weak cosmic censorship conjecture in BTZ black holes with scalar fields," Chin. Phys. C 44, no. 1, 015101 (2020) doi:10.1088/1674-1137/44/1/015101 arXiv:1812.03459 [gr$\mathrm{qc}]]$.

[53] X. X. Zeng and H. Q. Zhang, "Thermodynamics and weak cosmic censorship conjecture in Born-Infeld-anti-de Sitter black holes," Chin. Phys. C 45, no.2, 025112 (2021) doi:10.1088/1674-1137/abd088 arXiv:1901.04247 [hep-th]].

[54] D. Chen, W. Yang and X. Zeng, "Thermodynamics and weak cosmic censorship conjecture in Reissner-Nordström anti-de Sitter black holes with scalar field," Nucl. Phys. B 946, 114722 (2019) doi:10.1016/j.nuclphysb.2019.114722 arXiv:1901.05140 [hep-th]].

[55] B. Gwak, "Weak Cosmic Censorship with Pressure and Volume in Charged Anti-de Sitter Black Hole under Charged Scalar Field," JCAP 1908, 016 (2019) doi:10.1088/14757516/2019/08/016 [arXiv:1901.05589 [gr-qc]].

[56] X. X. Zeng, Y. W. Han and D. Y. Chen, "Thermodynamics and weak cosmic censorship conjecture of BTZ black holes in extended phase space," Chin. Phys. C 43, no. 10, 105104 (2019) doi:10.1088/1674-1137/43/10/105104 [arXiv:1901.08915 [gr-qc]].

[57] D. Chen, "Thermodynamics and weak cosmic censorship conjecture in extended phase spaces of anti-de Sitter black holes with particles' absorption,” Eur. Phys. J. C 79, no. 4, 353 (2019) doi:10.1140/epjc/s10052-019-6874-5 [arXiv:1902.06489 [hep-th]].

[58] P. Wang, H. Wu and H. Yang, "Thermodynamics of nonlinear electrodynamics black holes and the validity of weak cosmic censorship at charged particle absorption," Eur. Phys. J. C 79, no. 7, 572 (2019). doi:10.1140/epjc/s10052-019-7090-z

[59] X. X. Zeng, X. Y. Hu and K. J. He, "Weak cosmic censorship conjecture with pressure and volume in the Gauss-Bonnet AdS black hole," Nucl. Phys. B 949, 114823 (2019) doi:10.1016/j.nuclphysb.2019.114823 [arXiv:1905.07750 [hep-th]].

[60] W. Hong, B. Mu and J. Tao, "Thermodynamics and weak cosmic censorship conjecture in the charged RN-AdS black hole surrounded by quintessence under the scalar field," Nucl. Phys. B 949, 114826 (2019) doi:10.1016/j.nuclphysb.2019.114826 [arXiv:1905.07747 [gr-qc]].

[61] T. T. Hu, Y. Song, S. Sun, H. B. Li and Y. Q. Wang, "Weak cosmic censorship in Born-Infeld 
electrodynamics and bound on charge-to-mass ratio," Eur. Phys. J. C 80, no. 2, 147 (2020) doi:10.1140/epjc/s10052-020-7703-6 [arXiv:1906.00235 [hep-th]].

[62] S. Q. Hu, Y. C. Ong and D. N. Page, "No evidence for violation of the second law in extended black hole thermodynamics," Phys. Rev. D 100, no. 10, 104022 (2019) doi:10.1103/PhysRevD.100.104022 [arXiv:1906.05870 [gr-qc]].

[63] B. Mu and J. Tao, "Minimal Length Effect on Thermodynamics and Weak Cosmic Censorship Conjecture in anti-de Sitter Black Holes via Charged Particle Absorption," arXiv:1906.10544 [gr-qc].

[64] K. J. He, X. Y. Hu and X. X. Zeng, "Weak cosmic censorship conjecture and thermodynamics in quintessence AdS black hole under charged particle absorption," Chin. Phys. C 43, no. 12, 125101 (2019) doi:10.1088/1674-1137/43/12/125101 [arXiv:1906.10531 [gr-qc]].

[65] Q. Gan, G. Guo, P. Wang and H. Wu, "Strong cosmic censorship for a scalar field in a Born-Infeld-de Sitter black hole," Phys. Rev. D 100, no. 12, 124009 (2019) doi:10.1103/PhysRevD.100.124009 [arXiv:1907.04466 [hep-th]].

[66] X. X. Zeng and X. Y. Hu, "Thermodynamics and weak cosmic censorship conjecture with pressure in the rotating BTZ black holes," arXiv:1908.03845 [gr-qc].

[67] K. J. He, G. P. Li and X. Y. Hu, "Violations of the weak cosmic censorship conjecture in the higher dimensional $f(R)$ black holes with pressure," Eur. Phys. J. C 80, no.3, 209 (2020) doi:10.1140/epjc/s10052-020-7669-4 [arXiv:1909.09956 [hep-th]].

[68] S. Q. Hu, B. Liu, X. M. Kuang and R. H. Yue, "Revisiting black hole thermodynamics in massive gravity: charged particle absorption and shell of dust falling," Chin. Phys. C 44, 105107 (2020) doi:10.1088/1674-1137/abab8a arXiv:1910.04437 [gr-qc]].

[69] B. Gwak, "Weak Cosmic Censorship in Kerr-Sen Black Hole under Charged Scalar Field," JCAP 03, 058 (2020) doi:10.1088/1475-7516/2020/03/058 [arXiv:1910.13329 [gr-qc]].

[70] Q. Gan, P. Wang, H. Wu and H. Yang, "Strong Cosmic Censorship for a Scalar Field in an Einstein-Maxwell-Gauss-Bonnet-de Sitter Black Hole," Chin. Phys. C 45, no.2, 025103 (2021) doi:10.1088/1674-1137/abccaf [arXiv:1911.10996 [gr-qc]].

[71] W. Hong, B. Mu and J. Tao, "Testing the weak cosmic censorship conjecture in torus-like black hole under charged scalar field," Int. J. Mod. Phys. D 29, no.12, 2050078 (2020) doi:10.1142/S0218271820500789 [arXiv:2001.09008 [physics.gen-ph]].

[72] B. Gwak, "Thermodynamics in Rotating Anti-de Sitter Black Holes with Massive Scalar Field 
in Three Dimensions," Chin. Phys. C 44, no.12, 12 (2020) doi:10.1088/1674-1137/abb656 arXiv:2002.09729 [gr-qc]].

[73] Y. Zhang and S. Gao, "Testing cosmic censorship conjecture near extremal black holes with cosmological constants," Int. J. Mod. Phys. D 23, 1450044 (2014) doi:10.1142/S0218271814500448 [arXiv:1309.2027 [gr-qc]].

[74] J. W. York, Jr., "Black hole thermodynamics and the Euclidean Einstein action," Phys. Rev. D 33, 2092 (1986). doi:10.1103/PhysRevD.33.2092

[75] H. W. Braden, J. D. Brown, B. F. Whiting and J. W. York, Jr., "Charged black hole in a grand canonical ensemble," Phys. Rev. D 42, 3376 (1990). doi:10.1103/PhysRevD.42.3376

[76] S. Carlip and S. Vaidya, "Phase transitions and critical behavior for charged black holes," Class. Quant. Grav. 20, 3827 (2003) doi:10.1088/0264-9381/20/16/319 [gr-qc/0306054].

[77] A. P. Lundgren, "Charged black hole in a canonical ensemble," Phys. Rev. D 77, 044014 (2008) doi:10.1103/PhysRevD.77.044014 gr-qc/0612119.

[78] J. X. Lu, S. Roy and Z. Xiao, "Phase transitions and critical behavior of black branes in canonical ensemble," JHEP 1101, 133 (2011) doi:10.1007/JHEP01(2011)133 arXiv:1010.2068 [hep-th]].

[79] C. Wu, Z. Xiao and J. Xu, "Bubbles and Black Branes in Grand Canonical Ensemble," Phys. Rev. D 85, 044009 (2012) doi:10.1103/PhysRevD.85.044009 [arXiv:1108.1347 [hep-th]].

[80] J. X. Lu, R. Wei and J. Xu, "The phase structure of black D1/D5 (F/NS5) system in canonical ensemble," JHEP 1212, 012 (2012) doi:10.1007/JHEP12(2012)012 arXiv:1210.0708 [hepth]].

[81] J. X. Lu and R. Wei, "Modulating the phase structure of black D6 branes in canonical ensemble," JHEP 1304, 100 (2013) doi:10.1007/JHEP04(2013)100 arXiv:1301.1780 [hepth]].

[82] D. Zhou and Z. Xiao, "Phase structures of the black $\mathrm{D} p-\mathrm{D}(p+4)$-brane system in various ensembles I: thermal stability,” JHEP 1507, 134 (2015) doi:10.1007/JHEP07(2015)134 arXiv:1502.00261 [hep-th]].

[83] Z. Xiao and D. Zhou, "Phase structures of the black $\mathrm{D} p-\mathrm{D}(p+4)$-brane system in various ensembles II: electrical and thermodynamic stability," JHEP 1509, 028 (2015) doi:10.1007/JHEP09(2015)028 [arXiv:1507.02088 [hep-th]].

[84] P. Wang, H. Yang and S. Ying, "Thermodynamics and phase transition of a 
Gauss-Bonnet black hole in a cavity," Phys. Rev. D 101, no.6, 064045 (2020) doi:10.1103/PhysRevD.101.064045 [arXiv:1909.01275 [gr-qc]].

[85] N. Sanchis-Gual, J. C. Degollado, P. J. Montero, J. A. Font and C. Herdeiro, "Explosion and Final State of an Unstable Reissner-Nordstrom Black Hole," Phys. Rev. Lett. 116, no. 14, 141101 (2016) doi:10.1103/PhysRevLett.116.141101 [arXiv:1512.05358 [gr-qc]].

[86] N. Sanchis-Gual, J. C. Degollado, C. Herdeiro, J. A. Font and P. J. Montero, "Dynamical formation of a Reissner-Nordstrom black hole with scalar hair in a cavity," Phys. Rev. D 94, no. 4, 044061 (2016) doi:10.1103/PhysRevD.94.044061 arXiv:1607.06304 [gr-qc]].

[87] P. Basu, C. Krishnan and P. N. B. Subramanian, "Hairy Black Holes in a Box," JHEP 1611, 041 (2016) doi:10.1007/JHEP11(2016)041 arXiv:1609.01208 [hep-th]].

[88] Y. Peng, B. Wang and Y. Liu, "On the thermodynamics of the black hole and hairy black hole transitions in the asymptotically flat spacetime with a box," Eur. Phys. J. C 78, no. 3, 176 (2018) doi:10.1140/epjc/s10052-018-5652-0 [arXiv:1708.01411 [hep-th]].

[89] Y. Peng, "Studies of a general flat space/boson star transition model in a box through a language similar to holographic superconductors," JHEP 1707, 042 (2017) doi:10.1007/JHEP07(2017)042 [arXiv:1705.08694 [hep-th]].

[90] Y. Peng, "Scalar field configurations supported by charged compact reflecting stars in a curved spacetime," Phys. Lett. B 780, 144 (2018) doi:10.1016/j.physletb.2018.02.068 arXiv:1801.02495 [gr-qc]].

[91] Y. Peng, "Scalarization of compact stars in the scalar-Gauss-Bonnet gravity," JHEP 1912, 064 (2019) doi:10.1007/JHEP12(2019)064 arXiv:1910.13718 [gr-qc]].

[92] Y. Peng, "Analytical investigations on formations of hairy neutral reflecting shells in the scalar-Gauss-Bonnet gravity,” Eur. Phys. J. C 80, no.3, 202 (2020) doi:10.1140/epjc/s10052020-7778-0 [arXiv:2002.01892 [gr-qc]].

[93] P. Wang, H. Wu and H. Yang, "Thermodynamics and Phase Transition of a Nonlinear Electrodynamics Black Hole in a Cavity,” JHEP 1907, 002 (2019) doi:10.1007/JHEP07(2019)002 arXiv:1901.06216 [gr-qc]].

[94] K. Liang, P. Wang, H. Wu and M. Yang, "Phase structures and transitions of Born-Infeld black holes in a grand canonical ensemble," Eur. Phys. J. C 80, no.3, 187 (2020) doi:10.1140/epjc/s10052-020-7750-z arXiv:1907.00799 [gr-qc]].

[95] P. Wang, H. Wu and H. Yang, "Thermodynamic Geometry of AdS Black Holes and Black 
Holes in a Cavity," Eur. Phys. J. C 80, no.3, 216 (2020) doi:10.1140/epjc/s10052-020-7776-2 arXiv:1910.07874 [gr-qc]].

[96] F. Simovic and R. B. Mann, "Critical Phenomena of Charged de Sitter Black Holes in Cavities," Class. Quant. Grav. 36, no. 1, 014002 (2019) doi:10.1088/1361-6382/aaf445 arXiv:1807.11875 [gr-qc]].

[97] S. Haroon, R. A. Hennigar, R. B. Mann and F. Simovic, "Thermodynamics of Gauss-Bonnetde Sitter Black Holes,” Phys. Rev. D 101, 084051 (2020) doi:10.1103/PhysRevD.101.084051 arXiv:2002.01567 [gr-qc]].

[98] B. Mu, P. Wang and H. Yang, "Covariant GUP Deformed Hamilton-Jacobi Method," Adv. High Energy Phys. 2017, 3191839 (2017) doi:10.1155/2017/3191839 arXiv:1408.5055 [grqc]].

[99] J. Tao, P. Wang and H. Yang, "Black hole radiation with modified dispersion relation in tunneling paradigm: Static frame," Nucl. Phys. B 922, 346 (2017) doi:10.1016/j.nuclphysb.2017.06.022 arXiv:1505.03045 [gr-qc]].

[100] J. Jiang, B. Deng and Z. Chen, "Static charged dilaton black hole cannot be overcharged by gedanken experiments," Phys. Rev. D 100, no.6, 066024 (2019) doi:10.1103/PhysRevD.100.066024 arXiv:1909.02219 [hep-th]].

[101] J. Jiang, X. Liu and M. Zhang, "Examining the weak cosmic censorship conjecture by gedanken experiments for Kerr-Sen black holes," Phys. Rev. D 100, no.8, 084059 (2019) doi:10.1103/PhysRevD.100.084059 [arXiv:1910.04060 [hep-th]].

[102] X. Y. Wang and J. Jiang, "Examining the weak cosmic censorship conjecture of RNAdS black holes via the new version of the gedanken experiment," JCAP 07, 052 (2020) doi:10.1088/1475-7516/2020/07/052 [arXiv:1911.03938 [hep-th]]. 